\title{
Lie transformation on shortcut to adiabaticity in parametric driving quantum system
}

\author{
Jian-jian Cheng, Yao Du, and Lin Zhang* \\ School of physics and information technology, Shaanxi Normal University,Xi'an 710119, P. R. China
}

\begin{abstract}
Shortcut to adiabaticity (STA) is a speed way to produce the same final state that would result in an adiabatic, infinitely slow process. Two typical techniques to engineer STA are developed by either introducing auxiliary counterdiabatic fields or finding new Hamiltonians that own dynamical invariants to constraint the system into the adiabatic paths. In this paper, a consistent method is introduced to naturally connect the above two techniques with a unified Lie algebraic framework, which neatly removes the requirements of finding instantaneous states in the transitionless driving method and the invariant quantities in the invariant-based inverse engineering approach. The general STA schemes for different potential expansions are concisely achieved with the aid of this method.
\end{abstract}

PACS numbers: 03.65.Xp, 03.65.Fd, 42.50.Dv

\section{INTRODUCTION}

Quantum adiabatic technique is one of the most promising strategies for quantum computation based on quantum adiabatic theorem [1, 2. "Shortcuts to adiabaticity" (STA) are control protocols that take the system quickly to the same populations, or even the same final states reached by slowly adiabatic processes $[3$. A motivation to apply STA methods to quantum systems is to manipulate quantum states with a fast coherent dynamics with high fidelity [4]. Thus STA have become the typical techniques in preparing or driving internal and motional states in atomic, molecular, optical, and solidstate physics [5] $[$ ].

Due to the time consuming problem of slowly parametric driving in adiabatic evolution, two equivalent strategies for STA engineerings were developed so far, one is the transitionless quantum driving method [913], the other is the invariant-based inverse engineering technique 14, 15. The basic idea of transitionless quantum driving is to add an auxiliary interaction $\hat{H}_{1}(t)$ to reference Hamiltonian $\hat{H}_{0}(t)$,

$$
\hat{H}_{0}(t)=\sum_{n} \mid n(t) \varepsilon_{n}(t)\langle n(t)|,
$$

so that the dynamics follows exactly the approximate adiabatic evolution driven by $\hat{H}(t)$ starting from $\hat{H}_{0}(0)=$ $\sum_{n}|n(0)\rangle \varepsilon_{n}(0)\langle n(0)|$ through evolution operator

$$
\hat{U}(t)=\sum_{n}\left|\psi_{n}(t)\right\rangle\langle n(0)|,
$$

where

$$
\left|\psi_{n}(t)\right\rangle=e^{-\frac{i}{\hbar} \int_{0}^{t} d t^{\prime} \varepsilon_{n}\left(t^{\prime}\right)}|n(t)\rangle
$$

is the adiabatic states starting with $|n(0)\rangle$ without the geometric phase for the one parametric driving system.

*Electronic address: zhanglincn@snnu.edu.cn
The auxiliary interaction is then given by Berry's formulation [16].

$$
\begin{aligned}
\hat{H}(t) & =i \hbar\left(\partial_{t} \hat{U}\right) U^{\dagger} \\
& =\hat{H}_{0}(t)+i \hbar \sum_{n}\left|\partial_{t} n(t)\right\rangle\langle n(t)| \\
& \equiv \hat{H}_{0}(t)+\hat{H}_{1}(t)
\end{aligned}
$$

where $\hat{H}_{1}(t)$ is nondiagonal in the basis of $|n(t)\rangle$, and it requires a full knowledge of spectral properties of the instantaneous Hamiltonian $\hat{H}_{0}(t)$. However, in most practical cases, the auxiliary interaction calculated by instantaneous eigenstates are very hard to carry out. Now we give an alternative method to do STA in a general parametric driving system with Lie transformations. Starting from a general harmonic oscillator Hamiltonian [17, three independent generators can be separately defined by

$$
\hat{J}_{+}=\frac{1}{2 \hbar} \hat{x}^{2}, \hat{J}_{-}=\frac{1}{2 \hbar} \hat{p}^{2}, \hat{J}_{0}=\frac{i}{4 \hbar}(\hat{x} \hat{p}+\hat{p} \hat{x})
$$

where $\hat{J}_{0}$ is an anti-Hermitian operator for $\hat{J}_{0}^{\dagger}=-\hat{J}_{0}$. The three operators satisfy the $\mathrm{SU}(2)$ commutation relations

$$
\left[\hat{J}_{+}, \hat{J}_{-}\right]=2 \hat{J}_{0},\left[\hat{J}_{0}, \hat{J}_{ \pm}\right]= \pm \hat{J}_{ \pm}
$$

and we can introduce three independent unitary transformations based on $\mathrm{SU}(2)$ by [18,

$$
\hat{U}_{ \pm}(t)=e^{i \theta_{ \pm}(t) \hat{J}_{ \pm}}, \hat{U}_{0}(t)=e^{2 \theta_{0}(t) \hat{J}_{0}}
$$

where the transformation parameters $\theta_{ \pm}(t)$ and $\theta_{0}(t)$ are piecewise continuous real functions defined within a control time interval $[0, \tau]$. The above quantum transformations $\hat{U}_{ \pm}(t)$ and $\hat{U}_{0}(t)$ correspond to classical canonical transformations called rotations and squeezing, respectively. This type of unitary operators constitutes our "Lie transformations", which provides a general method to construct the adiabatic Hamiltonian for STA designs. 


\section{PARAMETRICALLY DRIVEN QUANTUM HARMONIC OSCILLATOR}

The first example is a fast harmonic trap expansion, which has been received much attentions because of its fundamental and practical implications. It applies to the cold atoms involving an adiabatic tuning of the system after a cooling phase [19]. For a parametric driven harmonic oscillator, we consider the system Hamiltonian

$$
\hat{H}_{0}(t)=\frac{\hat{p}^{2}}{2 m}+\frac{1}{2} m \omega^{2}(t) \hat{x}^{2}=\hbar \omega(t)\left(\hat{a}_{t}^{\dagger} \hat{a}_{t}+\frac{1}{2}\right),
$$

where $\hat{a}_{t}$ and $\hat{a}_{t}^{\dagger}$ are the annihilation and creation operators at time $t$ defined by

$$
\begin{aligned}
& \hat{a}_{t}=\sqrt{\frac{m \omega(t)}{2 \hbar}}\left[\hat{x}+\frac{i}{m \omega(t)} \hat{p}\right], \\
& \hat{a}_{t}^{\dagger}=\sqrt{\frac{m \omega(t)}{2 \hbar}}\left[\hat{x}-\frac{i}{m \omega(t)} \hat{p}\right] .
\end{aligned}
$$

Since the frequency depends on time, the instantaneous ladder operator $\hat{a}_{t}\left(\hat{a}_{t}^{\dagger}\right)$ will create (annihilate) different instantaneous states at different times adapting to different frequencies.

Now, we consider STA control on the parametric oscillator from $t=0$ to $\tau$ by Lie transformations. In this case, the squeezing operator can be written as

$$
\hat{U}_{0}(t)=e^{2(\ln r) \hat{J}_{0}}=e^{\frac{1}{2}(\ln r)\left[\hat{a}^{2}-\left(\hat{a}^{\dagger}\right)^{2}\right]},
$$

where the subscript $t$ of $\hat{a}$ and $\hat{a}^{\dagger}$ has been dropped because the squeezing combination $\hat{a}^{2}-\left(\hat{a}^{\dagger}\right)^{2}$ is actually independent of time. Here, the introduced transformation parameter $r(t)=\sqrt{\frac{\omega(t)}{\omega(0)}}$ plays as a linear squeezing (adiabatic) scaling factor, which gives (see Appendix IV)

$$
\hat{U}_{0}(t)|n(0)\rangle=|n(t)\rangle,
$$

and the instantaneous states of the final system satisfies

$$
\hat{H}_{0}(t)|n(t)\rangle=\varepsilon_{n}(t)|n(t)\rangle .
$$

A direct substitution of Eq. (12) into Eq.4 shows that the adiabatic Hamiltonian is

$$
\begin{aligned}
\hat{H}(t)= & \sum_{n} \hat{U}_{0}|n(0)\rangle \varepsilon_{n}(t)\langle n(0)| \hat{U}_{0}^{\dagger} \\
& +i \hbar \sum_{n} \dot{\hat{U}}_{0}|n(0)\rangle\langle n(0)| \hat{U}_{0}^{\dagger} \\
= & r^{2}(t) \hat{U}_{0} \hat{H}_{0}(0) \hat{U}_{0}^{\dagger}+i \hbar \dot{\hat{U}}_{0} \hat{U}_{0}^{\dagger} \\
= & \frac{\hat{p}^{2}}{2 m}+\frac{1}{2} m \omega^{2}(t) \hat{x}^{2}-\frac{\dot{r}}{2 r}(\hat{x} \hat{p}+\hat{p} \hat{x}),
\end{aligned}
$$

where the initial Hamiltonian $\hat{H}_{0}(0)=\frac{\hat{p}^{2}}{2 m}+\frac{1}{2} m \omega_{0}^{2} \hat{x}^{2}$ with $\omega_{0} \equiv \omega(0)$. Then the auxiliary Hamiltonian $\hat{H}_{1}(t)$ is given by

$$
\hat{H}_{1}(t)=-\frac{\dot{r}}{2 r}(\hat{x} \hat{p}+\hat{p} \hat{x})=-\frac{\dot{\omega}(t)}{4 \omega(t)}(\hat{x} \hat{p}+\hat{p} \hat{x}),
$$

which is firstly obtained by Berry's formula [20]. This method provides a way to design the adiabatic driving of Eq. (4) without resorting to the instantaneous states of $\hat{H}_{0}(t)$. The result indicates that the higher speed of the state evolution, the larger intensity of the required auxiliary field needs to recover the adiabatic evolution. The result clearly implies that, to an initial state described by a linear superposition $\Psi(0)=\sum_{n} c_{n}|n(0)\rangle$, the evolution generated by adiabatic Hamiltonian $\hat{H}(t)$ leads to adiabatic state $\Psi(t)=$ $\sum_{n} c_{n}\left|\psi_{n}(t)\right\rangle=\sum_{n} c_{n}|n(t)\rangle e^{-\frac{i}{\hbar} \int_{0}^{t} \varepsilon_{n}\left(t^{\prime}\right) d t^{\prime}}$.

However, the control field $\hat{H}_{1}(t)$ obtained by the above Lie transformation is still unpractical and hard to be realized because it is non-local. An alternative control field that is local and experimentally realizable can be established by a successive transform of

$$
\hat{U}_{+}(t)=e^{i \theta_{+}(t) \hat{J}_{+}}, \quad \theta_{+}(t)=-\frac{m \dot{r}(t)}{r(t)},
$$

which continues to transform Hamiltonian $\hat{H}(t)$ into

$$
\begin{aligned}
\hat{H}^{\prime}(t) & =\hat{U}_{+}(t) \hat{H}(t) \hat{U}_{+}^{\dagger}(t)+i \hbar \dot{\hat{U}}_{+}(t) \hat{U}_{+}^{\dagger}(t) \\
& =\frac{\hat{p}^{2}}{2 m}+\frac{1}{2} m \Omega^{2}(t) \hat{x}^{2}
\end{aligned}
$$

where

$$
\Omega^{2}(t)=\omega^{2}(t)-\frac{3}{4}\left[\frac{\dot{\omega}(t)}{\omega(t)}\right]^{2}+\frac{1}{2} \frac{\ddot{\omega}(t)}{\omega(t)}
$$

is the modified time-dependent frequency that can be easily realized in a practical control 21.

The new Hamiltonian $\hat{H}^{\prime}(t)$ has the same structure as the reference Hamiltonian $\hat{H}_{0}(t)$ but with different timedependent frequency, which can be formed by magnetic and optical fields in the cold atom experiments for a timevarying trapping.

Under the above successive transformations, the time evolution of the initial state $|n(0)\rangle$ is mapped to

$$
\left|\Phi_{n}(t)\right\rangle=\hat{U}_{+}(t)\left|\psi_{n}(t)\right\rangle=e^{-i \frac{m \dot{\omega}(t)}{4 \hbar \omega(t)} x^{2}}\left|\psi_{n}(t)\right\rangle,
$$

which generates a similar final state of an adiabatic process evolving slowly from $\hat{H}_{0}(0)$ to $\hat{H}_{0}(\tau)$ if the control frequency $\Omega(t)$ meets the boundary conditions

$$
\Omega(0)=\omega(0) \equiv \omega_{0}, \quad \Omega(\tau)=\omega(\tau) \equiv \omega_{\tau} .
$$

The above conditions lead to

$$
\dot{\omega}(0)=\dot{\omega}(\tau)=0, \quad \ddot{\omega}(0)=\ddot{\omega}(\tau)=0 .
$$


The zero of the first time-derivative means that $\left|\Phi_{n}(0, \tau)\right\rangle=\left|\psi_{n}(0, \tau)\right\rangle$ and the zero of the second one implies $\hat{H}^{\prime}(0, \tau)=\hat{H}_{0}(0, \tau)$.

In order to design the frequency $\Omega(t)$ to meet Eq. (21), a polynomial ansatz of time function for $\omega(t)$ is often used 14

$$
\omega(t)=\omega_{0}+10 \delta s^{3}-15 \delta s^{4}+6 \delta s^{5},
$$

where $\delta=\omega_{\tau}-\omega_{0}$ and $s=t / \tau$. The trajectories with

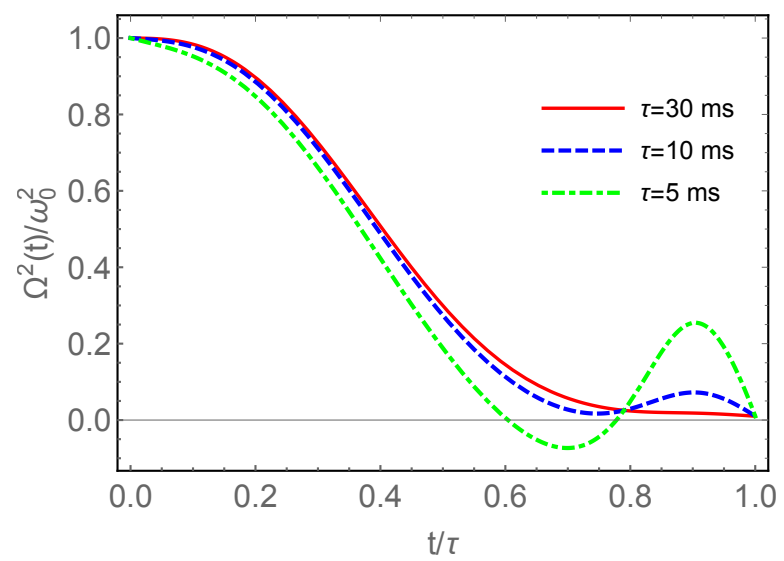

FIG. 1: The adiabatic drivings of the trap frequency $\Omega^{2}(t)$ changing from $\omega_{0}=2 \pi \times 250 \mathrm{~Hz}$ to $\omega_{\tau}=2 \pi \times 25 \mathrm{~Hz}$ are displayed for three different control times $\tau=30 \mathrm{~ms}$ (red solid line), $\tau=10 \mathrm{~ms}$ (blue dashed line) and $\tau=5 \mathrm{~ms}$ (green dot-dashed line).

different control times are displayed in Fig,1. We can see $\Omega^{2}(t)$ may become negative during some time intervals if the control process is very fast (green dot-dashed line), making the potential becomes an expulsive parabola. These state-independent shortcuts are ideal for maximal robustness owing to no excitation in the final state but allowing for excitations in the intermediate. With Lie transformation, it is convenient to verify that the above STA design is equivalent to the Lewis-Riesenfeld (LR) invariants method 14. If we set the total transformation $\hat{U}_{I}(t)=\hat{U}_{+}(t) \hat{U}_{0}(t)$, then the new adiabatic Hamiltonian $\hat{H}^{\prime}(t)$ is obtained by

$$
\hat{H}^{\prime}(t)=r^{2} \hat{I}(t)+i \hbar \dot{\hat{U}}_{I}(t) \hat{U}_{I}^{\dagger}(t),
$$

where the LR invariant is defined by

$$
\hat{I}(t)=\hat{U}_{I}(t) \hat{H}_{0}(0) \hat{U}_{I}^{\dagger}(t)
$$

Naturally, we can see $\hat{I}(0)=\hat{H}_{0}(0)$ and it satisfies

$$
\frac{d \hat{I}(t)}{d t}=\frac{\partial \hat{I}(t)}{\partial t}-\frac{1}{i \hbar}\left[\hat{H}^{\prime}(t), \hat{I}(t)\right]=0 .
$$

From Eq. 24, we can find the eigenvectors of $\hat{I}(t)$ are

$$
\left|\phi_{n}(t)\right\rangle=\hat{U}_{I}(t)|n(0)\rangle
$$

which satisfy

$$
\hat{I}(t)\left|\phi_{n}(t)\right\rangle=\lambda_{n}\left|\phi_{n}(t)\right\rangle
$$

where $\lambda_{n}=\left(n+\frac{1}{2}\right) \hbar \omega_{0}=\varepsilon_{n}(0)$. According to the invariant-based inverse engineering, the orthonormal eigenvectors of the invariant $\hat{I}(t)$ and the instantaneous eigenstates of system $\hat{H}_{0}(t)$ coincide at the initial and final times, then we naturally have

$$
\hat{I}(t)=\sum_{n}\left|\phi_{n}(t)\right\rangle \lambda_{n}\left\langle\phi_{n}(t)\left|=\sum_{n}\right| n(t)\right\rangle \lambda_{n}\langle n(t)|,
$$

which leads to

$$
\hat{I}(t)=r^{-2} \hat{H}_{0}(t)=\frac{\omega_{0}}{\omega(t)}\left[\frac{\hat{p}^{2}}{2 m}+\frac{1}{2} m \omega^{2}(t) \hat{x}^{2}\right] .
$$

As the new Hamiltonian of Eq. 23 must be equivalent to the Hamiltonian of the system $\hat{H}_{0}(t)$ at the initial and final times for STA controls, the boundary conditions will be

$$
\dot{\hat{U}}_{0}(t=0, \tau)=0, \dot{\hat{U}}_{+}(t=0, \tau)=0 \text {. }
$$

This new constraints can naturally recover Eq.21 and make $\hat{H}_{1}(t)$ vanish at the initial and final times. It should be noted that the transformed Hamiltonian $\hat{H}^{\prime}(t)$ we found here is just the Hamiltonian for LR dynamical invariant $\hat{I}(t)$. This is the essence of invariant-based inverse engineering and our method avoids the difficulty for constructing dynamical invariants $\hat{I}(t)$ for a given system 22 .

In conclusion, complementary to existing approaches about harmonic oscillator expansions, the Lie algebraic method proposed here provides a more general unified framework for STA contorls.

\section{POWER-LAW TRAP}

In the Lie algebraic framework, a generation to other trapping potentials is convenient and direct. We begin our trick with a free particle inside an expanding onedimensional box (quantum piston) [23]

$$
\hat{H}_{0}(t)=\frac{\hat{p}^{2}}{2 m}+V_{\mathrm{box}}(x, t),
$$

where the potential is

$$
V_{\text {box }}(x, t)=\left\{\begin{array}{cc}
0, & 0<x<L(t) \\
\infty, & \text { otherwise }
\end{array}\right.
$$

which describes an infinite square well with a moving boundary at $x=L(t)$. If the expansion is slow enough to follow adiabatic theorem, the exact solution can be 
expressed as

$$
\begin{aligned}
\psi_{n}(x, t) & =\sqrt{\frac{2}{L(t)}} \sin \left[\frac{n \pi x}{L(t)}\right] e^{-\frac{i}{\hbar} \int_{0}^{t} d t^{\prime} \frac{n^{2} \pi^{2} \hbar^{2}}{2 m L^{2}(t)}} \\
& \equiv \varphi_{n}\left[\frac{x}{L(t)}\right] e^{-\frac{i}{\hbar} \int_{0}^{t} \varepsilon_{n}\left(t^{\prime}\right) d t^{\prime}}
\end{aligned}
$$

where the instantaneous states satisfy

$$
\hat{H}_{0}(t) \varphi_{n}\left[\frac{x}{L(t)}\right]=\varepsilon_{n}(t) \varphi_{n}\left[\frac{x}{L(t)}\right] .
$$

Since the width of box depends on time, a squeezing transformation can be introduced

$$
\hat{U}_{0}(t)=e^{2 \ln \frac{L(0)}{L(t)} \hat{J}_{0}}
$$

with an initial width $L(0) \equiv L_{0}$. In the coordinate representation, the squeezing means increasing the wavelength of the free particle and reducing its momentum, which gives

$$
\hat{U}_{0}(t) \varphi_{n}\left(\frac{x}{L_{0}}\right)=\varphi_{n}\left[\frac{x}{L(t)}\right] .
$$

By using the same STA design method, we have

$$
\begin{aligned}
\hat{H}(t) & =\sum_{n} \hat{U}_{0}\left|\varphi_{n}\left(\frac{x}{L_{0}}\right)\right\rangle \varepsilon_{n}(t)\left\langle\varphi_{n}\left(\frac{x}{L_{0}}\right)\right| \hat{U}_{0}^{\dagger} \\
& +i \hbar \sum_{n} \dot{\hat{U}}_{0}\left|\varphi_{n}\left(\frac{x}{L_{0}}\right)\right\rangle\left\langle\varphi_{n}\left(\frac{x}{L_{0}}\right)\right| \hat{U}_{0}^{\dagger} \\
& =\frac{\hat{p}^{2}}{2 m}+\frac{\dot{L}(t)}{2 L(t)}(\hat{x} \hat{p}+\hat{p} \hat{x}) .
\end{aligned}
$$

The above Hamiltonian can be used to control quantum gas of noninteracting particles confined in an expanding box with the initial populations sampled microcanonically. The gas will remain a uniform distribution for an arbitrary expanding boundary $L(t)$ because the auxiliary term in Eq. (37) suppresses shock waves (excitations) by uniformly expanding the gas [24].

This technique can be easily generalized to all evenpower-law potentials 25]

$$
\hat{V}\left(r x_{1}, r x_{2}, \cdots, r x_{n}\right)=r^{k} \hat{V}\left(x_{1}, x_{2}, \cdots, x_{n}\right),
$$

with $k=2,4,6 \cdots$ due to the trapping properties of the potentials. Clearly, the case $k=2$ is the harmonic trapping we have considered in section II and $k \rightarrow \infty$ corresponds to the quantum box. The virial theorem shows that the average kinetic energy of the particle in the above potential $\bar{T}_{n}=\frac{k}{k+2} \bar{\varepsilon}_{n} \equiv \mu \bar{\varepsilon}_{n}[26]$.

For convenience, we set $L_{0}=1$ and the squeezing operator becomes $\hat{U}_{0}(t)=e^{-\mu \ln [L(t)] \hat{J}_{0}}$, which leads to

$$
\hat{U}_{0}(t) \varphi_{n}(x)=\varphi_{n}\left[\frac{x}{L^{\mu}(t)}\right],
$$

where $\varphi_{n}(x)$ are the wavefunctions of Hamiltonian with a one-dimensional potential of Eq.(38). Substitute Eq. (39) into Eq. (4), we can find the controlling adiabatic Hamiltonian

$$
\hat{H}(t)=\hat{H}_{0}(t)+\frac{k}{k+2} \frac{\dot{L}(t)}{2 L(t)}(\hat{x} \hat{p}+\hat{p} \hat{x}) .
$$

It shows that squeezing transformation greatly facilitates the STA designs for expansion or compression of the homogeneous trapping potentials. But still the auxiliary term is difficult to implement in the laboratory, we conduct another transformation $\hat{U}_{+}(t)=\exp \left[\frac{i m \dot{\xi}}{2 \hbar \xi} \hat{x}^{2}\right]$ with $\xi=L^{\mu}(t)$ on Eq. 40 and arrive

$$
\hat{H}^{\prime}(t)=\hat{H}_{0}(t)-\frac{1}{2} m \frac{k}{k+2} \frac{\ddot{L}(t)}{L(t)} x^{2} .
$$

The above new Hamiltonian introduces an auxiliary potential

$$
U_{\text {aux }}(x, t)=-\frac{1}{2} m \frac{k}{k+2} \frac{\ddot{L}(t)}{L(t)} x^{2}
$$

to control the system to a final adiabatic state. Now the auxiliary potential is a realistic harmonic potential which can be implemented in the laboratory with wellestablished technology and have been applied to perform a fast adiabatic squeezing in Bose-Einstein condensate 27 .

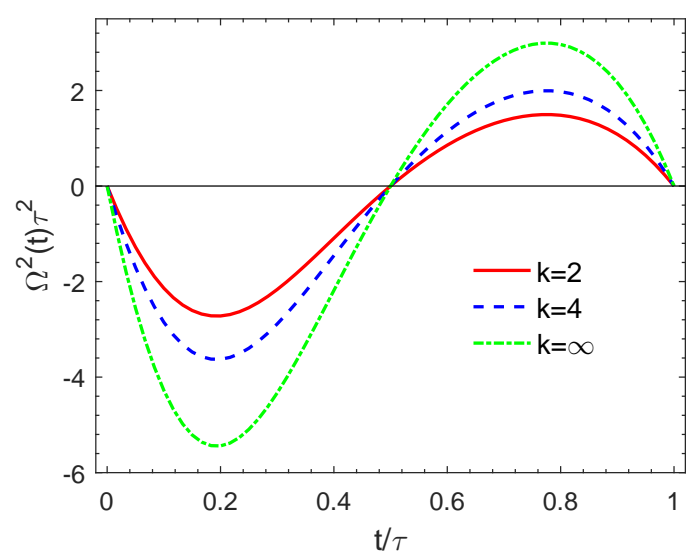

FIG. 2: Shortcuts to adiabaticity by adiabatic driving, for an expansion factor $L(\tau)=2$ with different power-law traps. The exponent values of auxiliary potentials correspond to harmonic oscillator (red solid line), a quartic trap potential (blue dashed line), and an expanding quantum piston (green dotdashed line).

In this case, the LR invariant in the inverse-engineering approach will also be defined by $\hat{I}(t)=\hat{U}_{I}(t) \hat{H}_{0}(0) \hat{U}_{I}^{\dagger}(t)$. Here, the effective frequency of the auxiliary potential, $\Omega^{2}(t)=-\frac{k}{k+2} \frac{\ddot{L}(t)}{L(t)}$, is the key to engineer the STA. According to Eq. 30, we impose the boundary conditions 
$\dot{L}(0, \tau)=\ddot{L}(0, \tau)=0$. A polynomial ansatz to meet the boundary condition can be designed by

$$
L(t)=1+[L(\tau)-1]\left[10 s^{3}+3 s^{4}(2 s-5)\right] .
$$

The driving frequencies $\Omega^{2}(t)$ for different power-law potentials are displayed in Fig 2. It shows a symmetric control process and $U_{\text {aux }}(x, t)$ performs a repulsive harmonic potential in an early stage $\left(t<\frac{\tau}{2}\right)$, achieving a speed-up STA in an arbitrary finite time $\tau$. In the subsequent stage $\left(t>\frac{\tau}{2}\right), \Omega^{2}(t)$ changes its sign to positive and $U_{\text {aux }}(x, t)$ becomes a trapping potential, slowing down the expanding mode. In other words, when the intermediate state deviates from the target state, the auxiliary potential gives a negative feedback, which reproduces the adiabatic result at the end of the evolution. Fig 2 also reveals that a stronger trapping potential means a higher auxiliary control energy. This conclusion has been considered in the expansion stroke of a quantum piston [28, where an auxiliary harmonic trap is introduced by an electromotive circuit which dissipates a mass of energy to preform the speed-up adiabatic Otto circle.

\section{CONCLUSION}

A unified algebraic framework is developed to design shortcuts to adiabaticity in both transitionless quantum driving and invariant-based inverse engineering method. We demonstrate that the two approaches is mathematically equivalent and share a common physical ground with Lie transformations, and provides a universal method to design STA for the general time-dependent parametric driving systems. It removes the demanding requirement to diagonalize the Hamiltonian of the system in the transitionless driving method, and the difficulty to find the invariant quantities in the invariantbased inverse engineering technique. The Lie transformation method present a powerful tool to design STA control in the homogenous power-law trapping system and can neatly connect the invariant-based method with the transitionless driving method by quickly diagonalizing the reference Hamiltonian. In summary, this work provides a deeper insight of shortcut-to-adiabaticity techniques in the quantum control problems.

\section{ACKNOWLEDGMENTS}

This work was supported by the National Natural Science Foundation of China for emergency management project (Grant No.11447025, 11847308).

\section{APPENDIX: THE DERIVATIONS OF SQUEEZING TRANSFORMATION}

The squeezing transformation is a unitary operator and easy to prove that

$$
\begin{aligned}
\hat{U}_{0}(t) \hat{a}_{0} \hat{U}_{0}^{\dagger}(t) & =\hat{a}_{0} \cosh (-\ln r)-\hat{a}_{0}^{\dagger} \sinh (-\ln r) \\
& =\sqrt{\frac{m \omega_{0}}{2 \hbar}}\left[\hat{x}+\frac{i}{m \omega_{0}} \hat{p}\right] \frac{1+r^{2}}{2 r} \\
& -\sqrt{\frac{m \omega_{0}}{2 \hbar}}\left[\hat{x}-\frac{i}{m \omega_{0}} \hat{p}\right] \frac{1-r^{2}}{2 r} \\
& =\sqrt{\frac{m \omega(t)}{2 \hbar}}\left[\hat{x}+\frac{i}{m \omega(t)} \hat{p}\right]=\hat{a}_{t},
\end{aligned}
$$

$$
\begin{aligned}
\hat{U}_{0}(t) \hat{a}_{0}^{\dagger} \hat{U}_{0}^{\dagger}(t) & =\hat{a}_{0}^{\dagger} \cosh (-\ln r)-\hat{a}_{0} \sinh (-\ln r) \\
& =\sqrt{\frac{m \omega_{0}}{2 \hbar}}\left[\hat{x}-\frac{i}{m \omega_{0}} \hat{p}\right] \frac{1+r^{2}}{2 r} \\
& -\sqrt{\frac{m \omega_{0}}{2 \hbar}}\left[\hat{x}+\frac{i}{m \omega_{0}} \hat{p}\right] \frac{1-r^{2}}{2 r} \\
& =\sqrt{\frac{m \omega(t)}{2 \hbar}}\left[\hat{x}-\frac{i}{m \omega(t)} \hat{p}\right]=\hat{a}_{t}^{\dagger},
\end{aligned}
$$

where $\hat{a}_{0} \equiv \hat{a}(0)$ and $\hat{a}_{t} \equiv \hat{a}(t)$. So that

$$
\begin{aligned}
\hat{U}_{0}(t)|n(0)\rangle & =\hat{U}_{0}(t) \frac{1}{\sqrt{n !}}\left(\hat{a}_{0}^{\dagger}\right)^{n}\left|0_{0}\right\rangle \\
& =\frac{1}{\sqrt{n !}} \hat{U}_{0}(t)\left(\hat{a}_{0}^{\dagger}\right)^{n} \hat{U}_{0}^{\dagger}(t) \hat{U}_{0}(t)\left|0_{0}\right\rangle \\
& =\frac{1}{\sqrt{n !}}\left[\hat{U}_{0}(t) \hat{a}_{0}^{\dagger} \hat{U}_{0}^{\dagger}(t)\right]^{n} \hat{U}_{0}(t)\left|0_{0}\right\rangle \\
& \equiv \frac{1}{\sqrt{n !}}\left(\hat{a}_{t}^{\dagger}\right)^{n}\left|0_{t}\right\rangle=|n(t)\rangle
\end{aligned}
$$

where the vacuum state at time $t$ is defined by $\left|0_{t}\right\rangle \equiv$ $\hat{U}_{0}(t)\left|0_{0}\right\rangle$.
[1] M. A. Nielsen and I. L. Chuang, Quantum Computation and Quantum Information (Cambridge University Press, Cambridge, England, 2000).

[2] E. Farhi, J. Goldstone, Sam Gutmann, Joshua Lapan,
Andrew Lundgren, Daniel Preda, A Quantum Adiabatic Evolution Algorithm Applied to Random Instances of an NP-Complete Problem, Science 292,472-475 (2001).

[3] D. Guéry-Odelin, A. Ruschhaupt, A. Kiely, E. Tor- 
rontegui, S. Martínez-Garaot, and J. G. Muga, Shortcuts to adiabaticity: Concepts, methods, and applications, Rev. Mod. Phys. 91, 045001 (2019).

[4] A. delCampo and K. Kim, Focus on Shortcuts to Adiabaticity, New J. Phys. 21, 050201 (2019).

[5] S. Masuda and K. Nakamura, Fast-Forward of Adiabatic Dynamics in Quantum Mechanics, Proc. R. Soc. A 466, 1135 (2009).

[6] S. Martinez-Garaot, E. Torrontegui. X. Chen, and J. G. Muga Fast transitionless expansion of cold atoms in optical Gaussian-beam traps, Phys. Rev. A 89, 053408 (2014).

[7] M. Palmero, E. Torrontegui,D. Guéry-Odelin, and J. G. Muga Fast atomic transport without vibrational heating, Phys. Rev. A 88, 053423 (2013).

[8] E. Torrontegui,S.Ibanez, Xi Chen,A. Ruschhaupt,D. Guéry-Odelin, and J. G. Muga Fast atomic transport without vibrational heating, Phys. Rev. A 83, 013415 (2011).

[9] M. Demirplak and S. A. Rice, Assisted Adiabatic Passage Revisited, J. Phys. Chem. B 109, 6838 (2005).

[10] M. Demirplak and S. A. Rice, On the Consistency, Extremal, and Global Properties of Counterdiabatic Fields, J. Chem. Phys. 129, 154111 (2008).

[11] S. Masuda and K. Nakamura, Acceleration of Adiabatic Quantum Dynamics in Electromagnetic Fields, Phys. Rev. A 84, 043434 (2011).

[12] M. V. Berry, Transitionless Quantum Driving, J. Phys. A: Math. Theor. 42, 365303 (2009).

[13] E. Torrontegui, S. Martínez-Garaot, A. Ruschhaupt, and J. G. Muga, Shortcuts to Adiabaticity: Fast-Forward Approach, Phys. Rev. A 86, 013601 (2012).

[14] X. Chen, A. Ruschhaupt, S. Schmidt, A. del Campo, D. Gury-Odelin, and J. G.Muga, Fast Optimal Frictionless Atom Cooling in Harmonic Traps: Shortcut to Adiabaticity, Phys. Rev. Lett. 104, 063002 (2010).

[15] A. Mostafazadeh, Dynamical Invariants, Adiabatic Approximation and the Geometric Phase (Nova, New York, 2001).

[16] X. Chen, E. Torrontegui, and J. G. Muga, LewisRiesenfeld Invariants and Transitionless Quantum Driv- ing, Phys. Rev. A 83, 062116 (2011).

[17] P. E. G. Assis and A. Fring, Non-Hermitian Hamiltonians of Lie algebraic type, J. Phys. A: Math. Theor. 42015203 (2009).

[18] L. Zhang, W. Zhang, Lie transformation method on quantum state evolution of a general time-dependent driven and damped parametric oscillator, Ann. Phys. 373424 (2016).

[19] Dionisis Stefanatos, Justin Ruths, and Jr-Shin Li Frictionless atom cooling in harmonic traps: A time-optimal approach, Phys. Rev. A 82, 063422 (2010).

[20] J G Muga, Xi Chen, S. Ibáñez, I Lizuain and A Ruschhaupt Transitionless quantum drivings for the harmonic oscillator, J. Phys. B: At. Mol. Opt. Phys. 43 085509 (2010).

[21] S. Ibáñez, Xi Chen, E. Torrontegui, J. G. Muga, and A. Ruschhaupt Multiple Schrödinger Pictures and Dynamics in Shortcuts to Adiabaticity, Phys. Rev. Lett. 109, 100403 (2012).

[22] E. Torrontegui, S. Martínez-Garaot and J. G. Muga Hamiltonian engineering via invariants and dynamical algebra, Phys. Rev.A 89, 043408 (2014)

[23] A. del Campo and M. G. Boshier, Shortcuts to Adiabaticity in a Time-Dependent Box, Sci. Rep. 2, 648 (2012).

[24] K. Nakamura, S. K. Avazbaev, Z. A. Sobirov, D. U. Matrasulov, and T. Monnai Ideal quantum gas in an expanding cavity: Nature of nonadiabatic force, Phys. Rev. E 83, 041133 (2011).

[25] M. V. Berry and G. Klein Newtonian trajectories and quantum waves in expanding force fields, J. Phys. A: Math. Gen.17 1805,1815 (1984)

[26] L. D. Landau and E. M. Lifshitz, Mechanics, 3rd ed. (Pergamon Press, Oxford) Sec.10,(1960)

[27] del Campo Fast frictionless dynamics as a toolbox for low-dimensional Bose-Einstein condensates, EPL 96, 60005 (2011).

[28] A. Tobalina, I. Lizuain and J. G. Muga Vanishing efficiency of a speeded-up ion-in-Paul-trap Otto engine, EPL 127, 20005 (2019). 